\title{
Critical Analysis of Diagnostic Methods in Pediatric Cardiology
}

\author{
Edmar A tik
}

São Paulo, SP - Brazil

Pediatric cardiology has recently experienced high advances in the diagnostic, therapeutical, and surgical fields, particularly in the first case, where confirmation of the clinical diagnosis to establish the patient's management is currently facilitated by increasingly sharp and even unquestionable images obtained through a variety of diagnostic methods ${ }^{1-6}$.

Due to this technological advance, clinical diagnosis has been more and more based on images obtained through echocardiography ${ }^{1-4}$, nuclear magnetic resonance imaging ${ }^{5}$, and even other methods ${ }^{6}$.

However, this excessive confidence in imaging may become a mistake when the clinical findings are not seen as guides for the functional diagnosis, which truly establishes the clinical and surgical therapeutical management.

This guidance depends essentially on the proper analysis of each type of clinical manifestation of heart disease, such as cyanosis, heart failure, and cardiac murmur.

Heart diseases expressed by marked cyanosis in the neonatal period usually have a pulmonary flow dependent on a patent ductus arteriosus and are represented by pulmonary atresia, tricuspid atresia, pulmonary valvar stenosis, tetralogy of Fallot, transposition of the great vessels, and other congenital heart diseases accompanied by pulmonary stenosis, including the complex anomalies. After the first month of life, cyanosis, usually more discrete, is due to heart diseases with a more accentuated pulmonary blood flow, such as tetralogy of Fallot.

Heart diseases expressed as heart failure are classified into 3 groups on a pathogenetic basis, as follows: 1) heart diseases depending on the persistence of pulmonary hypertension in Ebstein anomaly manifesting in the first hours of

Instituto do Coração do Hospital das Clínicas - FMUSP

Mailing address: Edmar Atik-InCor - Av. Dr. Enéas C. Aguiar 44 - 05403-000 São Paulo, SP, Brazil

English version by Stela Maris C. Gandour life; 2) heart diseases with obstruction of the left heart in which the systemic flow depends on the ductus arteriosus, such as coarctation of the aorta, aortic stenosis, and left heart hypoplasia, in the first week of life; 3 ) heart diseases with blood shunt depending on regression of pulmonary hypertension, such as, among the acyanotic types, ventricular septal defect, persistence of the ductus arteriosus, and defect of the atrioventricular septum; and, among the cyanotic types, common arterial trunk, total anomalous drainage of the pulmonary veins, and single ventricle.

In regard to heart diseases manifesting as cardiac murmur, we can cite acyanotic heart diseases of discrete repercussion, those with a left-to-right shunt, such as atrial and ventricular septal defects, and ductus arteriosus, and those with obstruction to the blood flow, such as pulmonary and aortic stenoses, and coarctation of the aorta.

In these types of clinical manifestations, the specific diagnostic characterization of each congenital heart disease becomes simple if we analyze the set of clinical findings, beginning with anamnesis and physical examination, complemented by radiographic and electrocardiographic findings.

The functional diagnosis obtained constitutes a basis for a more adequate guidance in regard to the management to be adopted, either the expecting clinical or the surgical type, which may occur on an emergency basis or in an elective form, intermingled with an improvement caused by effective therapy.

However, for confirming this diagnosis and choosing the most adequate management, echocardiography is currently an indispensable examination.

With echocardiography, we are able to characterize the diagnosis, both from the anatomical and functional points of view, in such an accurate manner that, undoubtedly, nowadays, the catheterization and angiographic studies have their uses limited for the same end.

The factors that have contributed to this methodological substitution relate to diagnostic accuracy, clinical and surgical confidence, and the good anatomical correlation provided by echocardiography. The advantages of this method are innumerable and include unquestionable efficien- 
cy, noninvasiveness, easy performance, and also the possibility of repetition whenever necessary, in order to eliminate occasional doubts raised by clinical findings.

Despite all these advances, because of the diagnostic limitation of the method, some situations require catheterization and angiographic studies for diagnostic confirmation. These situations may be divided into 2 groups with their respective subgroups: A) diagnostic difficulties of echocardiography: 1) limitation of the echocardiographic diagnosis; 2) clinical-echocardiographic contrast; B) diagnostic and therapeutical complementation: 1) hemodynamic assessment of pulmonary hypertension; 2) interventional therapeutic procedures; 3 ) electrophysiological studies; 4) endomyocardial biopsy.

In regard to limitations of the echocardiographic method, they may be summarized in the inadequate definition of certain cardiovascular structures, among which we may cite the following: anomalies of the pulmonary venous return associated with other defects, which cause decreased pulmonary blood flow, such as in complex heart diseases with pulmonary stenosis or pulmonary atresia, the systemic-pulmonary vessels and their respective anastomoses with the pulmonary arterial tree in pulmonary atresia and ventricular septal defect; the characterization of this pulmonary arterial tree in certain cases of tetralogy of Fallot accompanied by stenosis of one of the pulmonary arteries, or in genetic syndromes, such as Williams' syndrome, and congenital rubella; in addition, in arterial trajectories in anomalies of the aortic arch, in anomalies of the coronary arteries in their origin and trajectory, and in structural alterations with obstructions and aneurysms in congenital heart diseases, such as transposition of the great arteries and tetralogy of Fallot, or even in acquired heart diseases, such as Kawasaki's disease ${ }^{1-3}$.

Cardiol catheterization is required in situations in which clinical and echocardiographic diagnoses do not match for diagnostic characterization and establishment of the most adequate management.

Therefore, knowledge of the clinical features is fundamental for identifying some diagnostic mistakes on echocardiography. In many of these situations, the simple repetition of the echocardiographic study is enough to clarify diagnostic doubts. Some of these situations are described in the following paragraphs.

In anomalous origin of the coronary artery from the pulmonary arterial tree, the clinical findings are characteristic, but due to the sometimes peculiar trajectory simulating an origin from the left Valsalva sinus in an intramural aortic trajectory, the echocardiographic diagnosis is excessively difficult and confirmation requires a mandatory angiographic evaluation.

In an aortopulmonary window, whose clinical and echocardiographic manifestation is through mitral insufficiency with systolic murmur and a clear mitral reflux evaluated by Doppler, resulting from dilation of the mitral ring origina- ting from increased pulmonary blood flow, the angiographic study is also mandatory.

In marked pulmonary valvar stenosis or pulmonary atresia with right ventricular hypoplasia of variable degrees, angiography is also indicated in order to add diagnostic elements in regard to the real size of the ventricle.

In addition, in congenital heart diseases with multiple defects of systemic and pulmonary venous return, of the heart position, of absence of inferior vena cava with continuation through the azygos vein, angiography is required to program the most adequate technique in each case.

In regard to diagnostic and therapeutical complementation, formal indication of the hemodynamic study is mainly based not only on the necessary diagnosis but also on the prognostic dynamic evaluation of pulmonary hypertension, and determination of the use of vasodilators depending on the pharmacological response.

The several interventions at the level of valves and arterial obstructions that have been replacing cardiac surgery, mainly pulmonary valvar stenosis, aortic stenosis of the newborn infant, and coarctation of the aorta in children weighing more than $30 \mathrm{~kg}$, are also worthy of note.

Electrophysiological studies constitute another advance in hemodynamic diagnostic and mainly therapeutical complementation in ablation of anomalous bundles and atrioventricular ectopic foci.

Endomyocardial biopsy is currently fundamental in the diagnosis of an inflammatory process, both the myocardial infectious process and the rejection process in heart transplantation. It is undoubtedly important for diagnostic and therapeutical guidance.

Confirmation of clinical diagnosis has been performed by echocardiographic study that replaces cardiac catheterization, which has been oriented to the therapeutical intervention field, replacing cardiac surgery in many cardiac anomalies.

Therefore, according to estimates, corrective cardiac surgery has been performed in $85 \%$ of the cases with no previous hemodynamic study, based on the echocardiographic image ${ }^{5}$. Nuclear magnetic resonance imaging has lately added to this tendency, especially because its major application lies in the limitations of echocardiography already mentioned ${ }^{5}$.

However, I emphasize that despite the diagnostic advances, the clinical findings have not lost their importance. They constitute the basis for establishing the functional diagnosis, which is extremely important for choosing the right management, for refining other techniques through questioning and correlation of the findings, and for interacting with all diagnostic elements.

In conclusion, we can state that in the general management of heart diseases, the clinical findings are necessary and fundamental, and the imaging findings are unquestionably useful. 


\section{References}

1. Kaul S. New developments in ultrasound systems for contrast echocardiography. Clin Cardiol 1997; 20: 127-30.

2. Li J, Sanders SP. Three-dimensional echocardiography in congenital heart disease. Curr Opin Cardiol 1999; 14: 53-9.

3. Joffe II, Jacobs LE, Lampert C, Owen AA, Ioli AW, Kotler MN. Role of echocardiography in perioperative management of patients undergoing open heart surgery. Am Heart J 1996; 131: 162-76.
4. Randolph GR, Hagler DJ, Khandheria BK, et al. Remote telemedical interpretation of neonatal echocardiograms: impact on clinical management in a primary care setting. J Am Coll Cardiol 1999; 34: 241-5.

5. Davis CP, McKinnon GC, Debatin JF, von Schulthess GK. Ultra-high-speed MR imaging. Eur Radiol 1996; 6: 297-311.

6. Bengel FM, Schwaiger M. Nuclear medicine studies of the heart. Eur Radiol 1998;8: 1698-706. 